\title{
Application of Image Processing Technology Refined in The Medical Imaging
}

\author{
Fan Bing ${ }^{\mathrm{a}}$ \\ Nanyang Medical College, Nanyang, China
}

Keywords: image thinning, algorithm, Medical Imaging, edge analysis.

\begin{abstract}
Medical Imaging contains extremely rich information about the internal body of a patient in clinical diagnosis, new technology research and medical treatment, medical education and training as well as for the treatment of patients having an increasingly important role. Based on the medical imaging target edge image analysis, we presented an effective binary image thinning algorithm. The algorithm edge technology will feature the image information can be clearly and completely detected, and get a complete clear edge features refined image by thinning algorithm can greatly facilitate research studies next stage image. Experimental results show: With the refinement algorithm obtained image skeleton symmetry not only good, but also as a single pixel wide, and can maintain the original image intact connectivity.
\end{abstract}

\section{Background}

Digital image processing technology originated in the twenties, when using digital compression technology by cable sent the first one digital image to New York from London. 1964 US Jet Propulsion Laboratory JPL using the computer sent back to the satellite photos of the moon is processed to obtain a clear image ${ }^{[1]}$. Since then, space research, remote sensing, mapping and development in the field of medical imaging, digital image processing technology presents many new challenges, also contributed to the image processing technology to give attention to research and continuous development. Currently, digital image processing technology has become in many areas of research tools and objects, to promote the digital image processing and other disciplines of science communication $^{[2]}$. Medical image processing and analysis as an important application of digital image processing, and is closely related to people's physical and mental health, reflects the overall standard of living of citizens, so by medical workers, image processing technology research personnel, physical and electronic engineers, and many other fields researchers attention.

Image thinning means while maintaining the original image topology extraction process a singlepixel-wide skeleton as quickly as possible. Image Refinement is a digital image preprocessing an important part, whether it is or will play an important role in image analysis in image recognition ${ }^{[3]}$. Thus, the image thinning algorithm has been widespread concern in the field of image processing. In recent years, many scholars have proposed various thinning algorithm, there are two issues to consider in accordance with different angles, image thinning method: the edge points in the point and delete

\footnotetext{
${ }^{\mathrm{a}}$ Corresponding author : 305492086@qq.com
} 
reservations. Thinning algorithm based on the traditional edge points removed in the thinning process, only the edge points of the judgment can be deleted and dealt with accordingly ${ }^{[4]}$. Due to the impact study and tracking order neighborhood prone to asymmetric skeleton, thinning algorithm based on interior point is easy to make a reservation skeleton obtained more than one pixel. Based on the analysis of these two types of thinning algorithm proposed a new effective parallel thinning algorithm integrated use of these two refining methods.

\section{Image thinning processing}

Image thinning means to be refined from the image to get rid of unnecessary pixels, while maintaining the original edge feature information refining the original image frame. Image thinning algorithm is a digital image of a fundamental theory of the image can be substantially intact with the original image edge feature information it requires one to one feature information after thinning, in order to simplify the amount of data of the original image, increase the accuracy of the original image is used in place of the original image into the image processing or object recognition next stage ${ }^{[5]}$. Therefore, the quality of image thinning algorithm directly determines the next stage of image analysis or recognition of the quality of representation ${ }^{[6]}$. So, if the edge of the image feature information can be clearly and completely detected, and get a complete clear edge features refined image by thinning algorithm can greatly facilitate research studies next stage image. For example: after a phase of processing an image presentation description, image analysis, fingerprint analysis, object recognition, robot path planning, image compression information ${ }^{[7]}$. Therefore, image preprocessing stage is also thinning algorithm engineering application has a very wide range of applications. Generally before medical imaging image refinement must also the image pre-processing tasks such as image noise point removal, edge enhancement and other image points, this will help to minimize noise interference, thereby extracting clear and reliable edge point, and then refined ${ }^{[8]}$. Image refinement process usually has four steps: image de-noising; edge enhancement processing; edge extraction processing; edge positioning processing; image refinement. Its processing shown in Fig1.

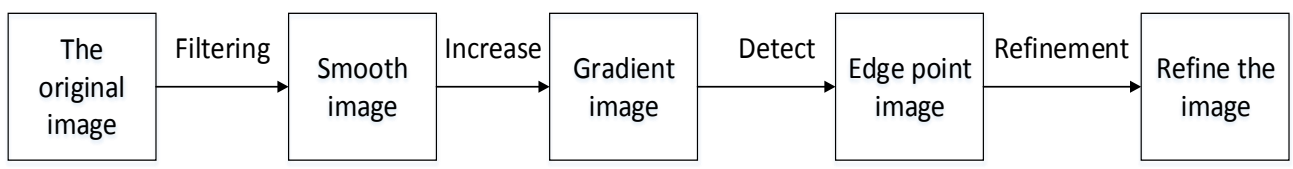

Figure 1. Image thinning process flowchart

\section{The image thinning algorithm design}

Any thinning algorithm generally must meet the following requirements: (1) skeleton image must maintain connectivity between the original image of the target edges. (2) skeleton image should be as far as possible the edge of the center line of the original image of the target. (3) it should be possible to refine the results obtained for a pixel width of the line image. (4) refinement faster. Accordingly, we propose a new, effective binary image thinning algorithm ${ }^{[9]}$. First, retention and special point within the image points must not be deleted. Next, remove the extra pixels. Finally, remove the excess branch lines.

(1) the feature point extraction method

Before application of the algorithm, we first obtain a binary image after thinning, for the binary image, we use eight-neighbor method to extract feature points.

This method is based on the refinement graph, each of them a spot before (i, j). (Ie fingerprint ridge line of black spots, denoted by 1 ; background recorded as 0 points) eight neighborhood (Figure 2) are processed to determine whether the point as a feature point. 


\begin{tabular}{|l|l|l|}
\hline $\mathrm{N}_{4}$ & $\mathrm{~N}_{3}$ & $\mathrm{~N}_{2}$ \\
\hline $\mathrm{N}_{5}$ & $(\mathrm{i}, \mathrm{j})$ & $\mathrm{N}_{1}$ \\
\hline $\mathrm{N}_{6}$ & $\mathrm{~N}_{7}$ & $\mathrm{~N}_{8}$ \\
\hline
\end{tabular} \begin{tabular}{|l|l|l|}
\hline 1 & 0 & 0 \\
\hline 0 & 1 & 1 \\
\hline 0 & 1 & 0 \\
\hline
\end{tabular}$\quad$\begin{tabular}{|l|l|l|l|}
\hline 0 & 1 & 0 \\
\hline 0 & 1 & 0 \\
\hline 0 & 0 & 0 \\
\hline
\end{tabular}

Figure 2. Select track points

Judgment is realized by the program, as follows:

If $\mathrm{N} 1+\mathrm{N} 2+\mathrm{N} 3+\mathrm{N} 4+\mathrm{N} 5+\mathrm{N} 6+\mathrm{N} 7+\mathrm{N} 8=\mathrm{O}$ was isolated points, otherwise:

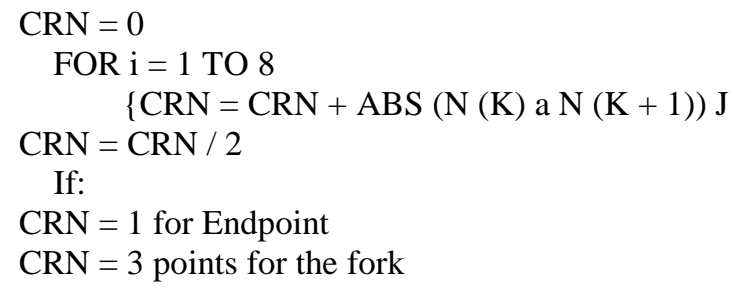

With the above rules, it can detect two types of endpoints and then point the feature point and its associated characteristic data into the feature point set.

(2) matching feature points

Although the image thinning pre pretreatment process through expansion and corrosion treatment to remove part of the cavity and burrs, but the final figure is still some refinement and from the point and the point of the fork formed by the burr hole formed and endpoint, obtained before the final feature points, it should be removed ${ }^{[10]}$. Detailed analysis of eight neighborhood and refinement Fig each point, not considerations rotation, and the formation of a total of three points, as shown in Figure 3 , where $(i, j)$ for the current and feature points, (1) (2) (3) eight were able to represent their neighborhood ridge extending direction of three black spots ${ }^{[11]}$.
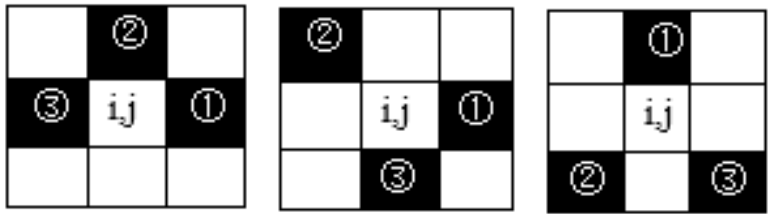

Figure 3. Three cases of the formation of the fork point

\section{Experimental results}

This paper presents an algorithm, pseudo feature points formed by the hollow, while removing burrs and short-term, concrete steps of the algorithm is as follows:

(1) For the feature point set AllMark [ ] in the current point; keep the number of black dots to find out if the group in three directions around the point to point on the fork

Pastpoint [ ], the decision points and set the current threshold and extending the number of steps, into the second step; if the endpoint, extending the number of steps set the threshold to enter the eighth step:

(2) along the current fork point determination extending forward looking extension line eight neighborhood of the current point is not pastpoint [ ] array of black spots; if this time has been three directions return value, the process proceeds to the seventh step; if currently extending step extends does not exceed the set threshold value and the current step size decision point is a feature point, if the endpoint, note the current extension step, and returns the endpoint related properties, credited Found [ ]; if they point to , and returns the associated properties of the point, which was credited Found [ ] in.

(4) If the current step extends does not exceed the threshold set extension step, and the black spots are not and neither end point, plus a number of steps will extend the extension line of the current point is stored pastpoint [] in, and Analyzing the current point to the point, go back to Step; 
(9) If the current decision point at the edge region of the fingerprint image is terminated earlier extension returns $(0,0,0)$, began to determine the next direction, return to step 2 ;

(6) exceeds the set step size, still can not find or fork end point, returns $(0,0,0)$; start determination under the direction of a return to the second step;

(7) obtained in three directions when the return value is returned if there is at least another point in both directions, and there are two identical fork point coordinates, you can determine the current judgment and point with these two points in turn returns are generated by empty pseudo-feature points, the two feature points are removed from the feature point set AllMark [ ]; if at least one direction returns the endpoint, and the endpoint is returned to the step count is less than the current determination fork point set Ae value, and that the current point and endpoint determination by the hair

Pseudo-generated feature prick, they are set from the feature point AllMark [ ] removed;

(8) extends forward from the current endpoint, if within the set threshold number of steps to find the other end, it is considered by both short-term isolation caused by the two endpoints are removed from AllMark []; the start determination AllMark [ ] the next feature point, go back one step.After the algorithm for image refinement results are as follows in Figure 4:
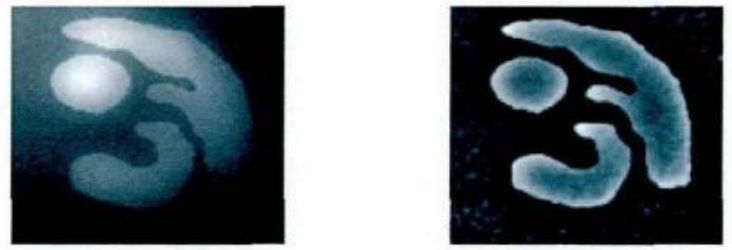

Figure 4.Before and after treatment in control

\section{References}

1. Tian Jie, Bao Shanglian, Zhou Ming-quan Medical Image Processing and Analysis Beijing: Electronic Industry Press, 2003.

2. Smith S. Overview of fMRI analysis. British Journal of Radiology, 2014, 77(2):167-175.

3. Zheng Yaqin, Tin Sum. Advances in medical image registration techniques. International Journal of Biomedical Engineering, 2006, (2) 40-44.

4. Antoniadis A., Bigot J., Sapatinas T. Wavelet estimators in nonparametric regression: a comparative simulation study. Journal of Statistical Software, 2001, pages 1-83.

5. Achim A., Bezerianos A. Tsakalides $P$ Novel Bayesian Multiscale Method for Speckle Removal in Medical Ultrasound Images. IEEE Transactions on Medical Imaging, 2001, 20(8):772-783.

6. Ma Liming, Li Yanting, Huang Zhaojia, other medical image processing technology [J] Modern Hospital Journal, 2007,7 (11): 15-19

7. Wang Shigeng, status quo and development direction of the medical image processing technology [J]. Medical Equipment, 2005,26 (12): 25-26.

8. Oleg S. Pianykh. Digital Imaging and Communications in Medicine (DICOM) [M]. Springer Berlin Heidelberg,2012.

9. Yong Cao, Xiaoguang Yue, Fei Xiong and Youjie Zhao. The Software Reliability Model Using Hybrid Model of Fractals and BP Neural Network. IETI Transactions on Computers. 2015.1(1):11-21

10. Qiao Zhihong, Wu Qing Qiao, Rain peak filtering algorithm based on direction of the fingerprint image enhancement Computer Engineering and Applications. 2004 (4): 87-89

11. A Ross, A K Jain, J Z Qian. Information fusion in biometrics. The third international conference on audio and video based biometric person authentication. 2012(4):354-360 